\title{
Finite groups with semi-subnormal Schmidt subgroups
}

\author{
V. N. Kniahina and V. S. Monakhov \\ Communicated by I. Ya. Subbotin
}

For the 70th anniversary of L. A. Kurdachenko

\begin{abstract}
A BSTRACT. A Schmidt group is a non-nilpotent group in which every proper subgroup is nilpotent. A subgroup $A$ of a group $G$ is semi-normal in $G$ if there exists a subgroup $B$ of $G$ such that $G=A B$ and $A B_{1}$ is a proper subgroup of $G$ for every proper subgroup $B_{1}$ of $B$. If $A$ is either subnormal in $G$ or is semi-normal in $G$, then $A$ is called a semi-subnormal subgroup of $G$. In this paper, we establish that a group $G$ with semi-subnormal Schmidt $\{2,3\}$-subgroups is 3 -soluble. Moreover, if all 5 -closed Schmidt $\{2,5\}$ subgroups are semi-subnormal in $G$, then $G$ is soluble. We prove that a group with semi-subnormal Schmidt subgroups is metanilpotent.
\end{abstract}

\section{Introduction}

All groups in this paper are finite. A Schmidt group is a non-nilpotent group in which every proper subgroup is nilpotent. These groups were first considered by O. Y. Schmidt [1]. He proved that a Schmidt group is biprimary, one of its Sylow subgroups is normal and another one is cyclic. Reviews about the structure of Schmidt groups and their applications in the theory of finite groups are available in [2], [3].

Since every non-nilpotent group contains a Schmidt subgroup, Schmidt groups are universal subgroups of finite groups. So the properties of

2010 MSC: 20E28, 20E32, 20E34.

Key words and phrases: finite soluble group, Schmidt subgroup, semi-normal subgroup, subnormal subgroup. 
Schmidt subgroups contained in a group have a significant influence on the group structure. Groups with some restrictions on Schmidt subgroups were investigated in many papers. For example, groups with subnormal Schmidt subgroups were studied in [4]-[5], and groups with Hall Schmidt subgroups were described in [6].

A subgroup $A$ of a group $G$ is semi-normal in $G$ if there exists a subgroup $B$ of $G$ such that $G=A B$ and $A B_{1}$ is a proper subgroup of $G$ for every proper subgroup $B_{1}$ of $B$. Obviously, every subgroup of prime index is semi-normal. Also a quasi-normal subgroup (i. e. a subgroup of $G$, that permutes with all subgroups of $G$ ) is semi-normal. In the simple group $\operatorname{PSL}(2,5)$ a subgroup $A$ which is isomorphic to the alternating group $A_{4}$ is a semi-normal Schmidt subgroup, but $A$ is not quasi-normal and not subnormal.

Some properties of semi-normal subgroups were obtained in [7]-[9]. The criteria for solubility of a group with some semi-normal Schmidt subgroups were established in [10].

We introduce the following concept, which combines subnormality and semi-normality.

Definition. A subgroup $A$ of a group $G$ is called semi-subnormal in $G$ if $A$ is either subnormal in $G$ or semi-normal in $G$.

In this paper, we establish that a group $G$ with semi-subnormal Schmidt $\{2,3\}$ - subgroups is 3 -soluble. Moreover, if all 5 -closed Schmidt $\{2,5\}$ subgroups are semi-subnormal in $G$, then $G$ is soluble. We prove that a group with semi-subnormal Schmidt subgroups is metanilpotent.

\section{Preliminary results}

The terminology in the article as in [11]-[12]. We write $Y \leqslant X$ if $Y$ is a subgroup of a group $X$. Recall that $A^{G}=\left\langle A^{g} \mid g \in G\right\rangle$ is the subgroup generated by all subgroups of $G$ that are conjugate to $A$. A group with a normal Sylow $p$-subgroup is called $p$-closed. Let $\pi$ be a set of primes. We say $G$ is a $\pi$-group if every prime divisor of $|G|$ lies in $\pi$. Let us agree to call the $S_{\langle p, q\rangle}$-group a Schmidt group with a normal Sylow $p$-subgroup and a cyclic Sylow $q$-subgroup.

Lemma 1 ([11, 2.41;2.43;5.31]). Let $H$ be a subnormal subgroup of a group $G$.

(1) If $U \leqslant G$, then $U \cap H$ is subnormal in $U$. In particular, if $H \leqslant$ $V \leqslant G$, then $H$ is subnormal in $V$.

(2) If $N$ is a normal subgroup of $G$, then $H N / N$ is a subnormal subgroup of $G / N$. 
(3) If $K$ is a subnormal subgroup of $G$, then $H \cap K$ and $\langle H \cup K\rangle$ are subnormal subgroups of $G$.

(4) $\pi(H)=\pi\left(H^{G}\right)$.

(5) If $\mathfrak{X}$ is a Fitting class and $H \in \mathfrak{X}$, then $H^{G} \in \mathfrak{X}$.

Lemma 2. If $X$ is a subnormal $S_{\langle p, q\rangle}$-subgroup of a group $G$, then $X^{G}$ is a $p$-closed $\{p, q\}$-subgroup.

Proof. Lemma 1 (4) implies that $X^{G}$ is a $\{p, q\}$-subgroup. The class of all $p$-closed subgroups is a Fitting class $[11,5.3]$. By Lemma $1(5), X^{G}$ is $p$-closed.

Lemma 3 ([4, Lemma 2]). If $K$ and $D$ are subgroups of a group $G$, $D$ is normal in $K$ and $K / D$ is an $S_{\langle p, q\rangle}$-subgroup, then each minimal supplement $L$ to the subgroup $D$ in $K$ has the following properties:

(1) $L$ is a p-closed $\{p, q\}$-subgroup;

(2) all proper normal subgroups of $L$ are nilpotent;

(3) $L$ contains an $S_{\langle p, q\rangle}$-subgroup $[P] Q$ such that $Q$ is not contained in $D$, and $L=([P] Q)^{L}=Q^{L}$.

Lemma 4 ([13, Lemma 1.6]). If $H$ is a subgroup of a soluble group $G$ and $|G: H|$ is a prime number, then $G / H_{G}$ is supersoluble.

Here $H_{G}=\cap_{x \in G} H^{x}$ is the core of $H$ in $G$.

The Frattini and Fitting subgroups of a group $G$ are denoted by $\Phi(G)$ and $F(G)$ respectively. The intersection of all normal subgroups of $G$, whose quotient groups belong to $\mathfrak{F}$ is called $\mathfrak{F}$-residual of $G$ and denoted by $G^{\mathfrak{F}}$.

Lemma 5 ([12, 24.2; 24.3]). If $\mathfrak{F}$ is a saturated formation, $G$ is a soluble minimal non-F-group, then the following statements hold:

(1) $G^{\mathfrak{F}}$ is a p-group for some $p \in \pi(G)$;

(2) $G^{\mathfrak{F}} / \Phi\left(G^{\mathfrak{F}}\right)$ is a chief factor of $G$;

(3) $G^{\mathfrak{F}} \Phi(G)=F(G)$.

Lemma 6. If $G$ is a soluble minimal non-metanilpotent group, then $G / F(G)$ is a Schmidt group.

Proof. The class of all metanilpotent groups is a saturated hereditary formation $\left[12\right.$, p. 36] and coincides with the product $\mathfrak{N N}=\mathfrak{N}^{2}$. First, let $\Phi(G)=1$. According to Lemma $5(3), G^{\mathfrak{N}^{2}}=F(G)$. Since $G^{\mathfrak{N}^{2}}$ is a $p$-group for some $p \in \pi(G)$ by Lemma $5(1)$, we have $F(G)=O_{p}(G)$. Since $G \notin \mathfrak{N}^{2}$, it follows that $G / F(G) \notin \mathfrak{N}$. 
Let $U / F(G)$ be a proper subgroup of $G / F(G)$. Then $U$ is metanilpotent and $U / F(U)$ is nilpotent. Since $G$ is soluble, we obtain $O_{p}(G)=F(G) \leqslant U$, it implies

$C_{G}(F(G))=Z(F(G)), \quad O_{p^{\prime}}(U)=1, \quad O_{p}(G)=F(G) \leqslant F(U)=O_{p}(U)$.

Let $H / F(G)=F(G / F(G))$. Then $H / F(G)$ is a $p^{\prime}$-subgroup and

$$
H \cap O_{p}(U)=O_{p}(G)=F(G) .
$$

Since $G / H$ is nilpotent, $U H / H \simeq U / U \cap H$ and $U / O_{p}(U)$ are nilpotent. So

$$
U / U \cap H \cap O_{p}(U)=U / F(G)
$$

is nilpotent. Therefore, all proper subgroups of $G / F(G)$ are nilpotent and $G / F(G)$ is a Schmidt group.

Let $\Phi(G) \neq 1$. According to Lemma $5(3)$, the subgroup $F(G)=$ $G^{\mathfrak{N}^{2}} \Phi(G)$. Since $\mathfrak{N}^{2}$ is a hereditary formation, we have $G / \Phi(G) \notin \mathfrak{N}^{2}$ and $G / \Phi(G)$ is a minimal non- $\mathfrak{N}^{2}$-group. By the hypothesis, $G$ is soluble, so $F(G / \Phi(G))=F(G) / \Phi(G)$ by [11, 4.21], and

$$
G / F(G) \simeq(G / \Phi(G)) /(F(G) / \Phi(G))=(G / \Phi(G)) /(F(G / \Phi(G)) .
$$

Since $\Phi(G / \Phi(G))=1$, by the above $(G / \Phi(G)) / F(G / \Phi(G))$ is a Schmidt group, therefore $G / F(G)$ is a Schmidt group.

Example 1. In the simple group $\operatorname{PSL}(2,5)$, all its proper subgroups are metanilpotent, they are even metabelian. Therefore, the condition of solubility of a group in Lemma 6 is not superfluous.

\section{Properties of semi-subnormal subgroups}

Lemma 7. (1) If $H$ is a semi-subnormal subgroup of a group $G$ and $H \leqslant X \leqslant G$, then $H$ is semi-subnormal in $X$.

(2) If $H$ is a semi-subnormal subgroup of a group $G, N$ is a normal subgroup of $G$, then $H N$ is semi-subnormal in $G$, and $H N / N$ is semisubnormal in $G / N$.

(3) If $H$ is a semi-subnormal subgroup of a group $G$ and $Y$ is nonempty set of elements in $G$, then the subgroup

$$
H^{Y}=\left\langle H^{y} \mid y \in Y\right\rangle
$$

is semi-subnormal in $G$. In particular, $H^{g}$ is semi-subnormal in $G$ for any $g \in G$. 
Proof. Suppose that $H$ is a subnormal subgroup of a group $G$. Then it follows from Lemma 1 that statements (1)-(3) are true. If $H$ is semi-normal in $G$, then assertions (1)-(3) are proved in [10, Lemma 2.5].

Proposition 1. Let $A$ be a semi-subnormal subgroup of a group $G$. Then the following statements holds.

(1) If $A$ is a 2-nilpotent subgroup, then $A^{G}$ is soluble.

(2) If $A$ is soluble and 3 does not divide the order of $A$, then $A^{G}$ is soluble.

(3) Let $p$ be the smallest prime divisor of the order of G. If $p$ does not divide the order of $A$, then $p$ does not divide the order of $A^{G}$. In particular, if $A$ is a subgroup of odd order, then $A^{G}$ is of odd order.

Proof. If $A$ is a semi-normal subgroup of $G$, then all three statements are known [10, lemmas 10 and 11]. If $A$ is a subnormal subgroup of $G$, then all three statements follow from Lemma $1(4-5)$.

Proposition 2. If $A$ is a semi-subnormal subgroup of a soluble group $G$ and $r$ is the largest prime from $\pi(G)$, then $O_{r}(A) \leqslant O_{r}(G)$.

Proof. It suffices to prove that $O_{r}(A)$ is subnormal in $G$. If $A$ is a subnormal subgroup of $G$, then $O_{r}(A)$ is subnormal in $G$ and $O_{r}(A) \leqslant O_{r}(G)$ by Lemma 1(5). Let $A$ be a semi-normal subgroup of $G$ and $Y$ be a minimal supplement to $A$ in $G$ such that $A$ is permutable with all subgroups of $Y$. We apply induction on the order of the group. Since $Y$ is soluble, there is a subgroup $X$ of prime index i.e $|Y: X|=t$ is a prime number. By the hypothesis, $A$ is permutable with $X$. Besides $A$ is a semi-subnormal subgroup of $A X$ by Lemma 7 (1). By induction, $O_{r}(A)$ is subnormal in $A X$. If $A X$ is normal in $G$, then $O_{r}(A)$ is subnormal in $G$. Suppose that $A X$ is not normal in $G$, i. e. $A X \neq(A X)_{G}$. Since $G=A Y$ and $A X<G$, we have

$$
1 \neq|G: A X|=\frac{|A||Y|}{|A \cap Y|}: \frac{|A||X|}{|A \cap X|}=\frac{|Y: X|}{|A \cap Y: A \cap X|}=t .
$$

By Lemma $4, G /(A X)_{G}$ is supersoluble and is isomorphic to a subgroup of the symmetric group $S_{t}$ of degree $t$. Since

$$
t=|G: A X|=\left|G /(A X)_{G}: A /(A X)_{G}\right|,
$$

then $t$ does not divide $\left|A X /(A X)_{G}\right|$. If $t=r$, then $A X /(A X)_{G}$ is a $r^{\prime}$ group. Hence

$$
O_{r}(A) \leqslant O_{r}(A X) \leqslant(A X)_{r} \leqslant(A X)_{G}
$$


and $O_{r}(A)$ is subnormal in $G$. If $t \neq r$, then $t<r$ and $G /(A X)_{G}$ is a $r^{\prime}$-group. Therefore, $A_{r} \leqslant(A X)_{G}$ and again $O_{r}(A)$ subnormal in $G$. Consequently, $O_{r}(A) \leqslant O_{r}(G)$.

\section{Semi-subnormal Schmidt subgroups}

Lemma 8. Suppose that all $S_{\langle p, q\rangle}$-subgroups of a group $G$ are semisubnormal in $G$.

(1) If $H$ is a subgroup of $G$, then all $S_{\langle p, q\rangle^{-}}$subgroups of $H$ are semisubnormal in $H$.

(2) If $N$ is a normal subgroup of $G$, then all $S_{\langle p, q\rangle}$ - subgroups of $G / N$ are semi-subnormal in $G / N$.

Proof. 1. The statement follows from Lemma 7 (1).

2. Let $S / N$ be an $S_{\langle p, q\rangle}$-subgroup of $G / N$ and $L$ be a minimal subgroup of $S$ such that $S=L N$. By Lemma 3, $L$ contains an $S_{\langle p, q\rangle}$-subgroup $A$ such that $L=A^{L}$. By the hypothesis, $A$ is semi-subnormal in $G$, and by Lemma 7 (3), $L$ is semi-subnormal in $G$. Now by Lemma 7 (2), the subgroup $L N / N=S / N$ is semi-subnormal in $G / N$.

Theorem 1. Let $A$ be a semi-subnormal Schmidt subgroup of a group $G$.

(1) If $A^{G}$ is insoluble, then $A / Z(A) \simeq A_{4}$.

(2) If $A^{G}$ is a simple group, then $A^{G} \simeq \operatorname{PSL}(2,5)$.

Proof. If $A$ is a subnormal subgroup of $G$, then $\pi\left(A^{G}\right)=\pi(A)$ by Lemma 2, so $A^{G}$ is soluble. Further, we assume that $A$ is a semi-normal subgroup of $G$. If $A^{G}$ is insoluble, then $A$ is a 2-closed $\{2,3\}$-subgroup according to [10, Theorem 1]. We deduce from the properties of Schmidt groups [3, Theorem 1.2] that $A / Z(A) \simeq A_{4}$. Let $A^{G}$ be a simple group. By Lemma 7 , the subgroup $A$ is semi-normal in $A^{G}$ and $\left|A^{G}: A\right|$ is a prime number by [10, Lemma 7]. Now $Z(A)=1$ by [14, V.7.2] and $A \simeq A_{4}$. In view of [[15], Theorem 1], we obtain that $A^{G} \simeq P S L(2,5)$.

Theorem 2. If all $\{2,3\}$-subgroups of a group $G$ are semi-subnormal in $G$, then $G$ is 3-soluble.

Proof. We use induction on the order of $G$. Let $N$ be a normal subgroup of $G$. By Lemma 8, all Schmidt $\{2,3\}$-subgroups are semi-subnormal in $N$ and in $G / N$. If $G \neq N \neq 1$, then by induction, $N$ and $G / N$ are 3 -soluble. This implies that $G$ likewise is 3 -soluble. We will therefore assume that $G$ is simple, in particular there are no subnormal Schmidt 
subgroups. Consequently, all Schmidt $\{2,3\}$-subgroups are semi-normal in $G$. According to [10, Theorem 2], $G$ is 3-soluble.

Corollary 1. If all Schmidt $\{2,3\}$-subgroups and all 5-closed Schmidt $\{2,5\}$-subgroups of a group $G$ are semi-subnormal, then $G$ is soluble.

In Corollary 1, three types of Schmidt subgroups: $S_{\langle 2,3\rangle}$-subgroups, $S_{\langle 3,2\rangle}$-subgroups, and $S_{\langle 5,2\rangle}$-subgroups should be semi-subnormal. The following examples of simple groups show that the condtion of semisubnormality of all these types of Schmidt subgroups is not superfluous.

Example 2. In $P S L\left(2,3^{3}\right)$, there are no $S_{\langle 5,2\rangle}$-subgroups and $S_{\langle 3,2\rangle^{-}}$ subgroups [16]; so the condition of being semi-subnormal for $S_{\langle 2,3\rangle}$-subgroups is not superfluous.

Example 3. In $S L(2,8)$, there are no $S_{\langle 5,2\rangle}$-subgroups and $S_{\langle 2,3\rangle}$-subgroups [16]; so the groups with semi-subnormal Schmidt $S_{\langle 5,2\rangle}$-subgroups and $S_{\langle 2,3\rangle}$-subgroups may be insoluble and the condition of being semisubnormal for $S_{\langle 3,2\rangle}$-subgroups is not superfluous.

Example 4. In $S z(8)$, there are no $\{2,3\}$-subgroups [16]; so the groups with semi-subnormal Schmidt $\{2,3\}$-subgroups may be insoluble and the condition of being semi-subnormal for $S_{\langle 5,2\rangle}$-subgroups is not superfluous.

Theorem 3. If all Schmidt subgroups of a group $G$ are semi-subnormal in $G$, then $G$ is metanilpotent.

Proof. By Corollary 1, $G$ is soluble. We use induction on the order of $G$. According to Lemma 8, in each proper subgroup and in each quotient group of $G$ different from $G$, all Schmidt subgroups are semi-subnormal. By induction, they are metanilpotent. The class of all metanilpotent groups is a hereditary saturated formation. By [17, Lemma 8], $G$ is primitive, and:

$$
G=[N] M, M<\cdot G, N=F(G)=O_{p}(G)=C_{G}(N),
$$

for some $p \in \pi(G)$. According to Lemma 6, the quotient group $G / N \simeq M$ is a Schmidt group. By the hypothesis, $M$ is either subnormal in $G$ or semi-normal in $G$. If $M$ is subnormal in $G$, then $M$ is normal in $G$ and $M \leqslant C_{G}(N)=N$, a contradiction. If $M$ is semi-normal in $G$, then $\mid G$ : $M|=| N \mid=p$ is a prime number by [10, Lemma 7]. Hence $G$ is supersoluble and therefore metanilpotent. 


\section{References}

[1] O. Y. Schmidt, Groups whose all subgroups are special, Matem. Sb., Vol. 31, 1924, P. 366-372 (in Russian).

[2] N. F. Kuzennyi, S. S. Levishchenko, Finite Shmidt's groups and their generalizations, Ukrainian Math. J., Vol. 43 (7-8), 1991, P. 898-904.

[3] V.S. Monakhov, The Schmidt groups, its existence and some applications, Tr. Ukrain. Math. Congr.-2001. Kiev: 2002, Section 1. P. 81-90 (in Russian).

[4] V. N. Kniahina, V.S. Monakhov, On finite groups with some subnormal Schmidt subgroups, Sib. Math. J., Vol. 45 (6), 2004, P. 1075-1079.

[5] V.A. Vedernikov, Finite groups with subnormal Schmidt subgroups, Algebra and Logic, Vol. 46 (6), 2007, P. 363-372.

[6] V. N. Kniahina, V.S. Monakhov, Finite groups with Hall Schmidt subgroups, Publ. Math. Debrecen, Vol. 81 (3-4), 2012, P. 341-350.

[7] V.V. Podgornaya, Semi-normal subgroups and supersolubility of finite groups, Vesti Nats.Akad. Navuk Belarusi. Ser. Fiz.-Mat. navuk. Vol. 4, 2000. P. 22-25. [in Russian]

[8] V.S. Monakhov, Finite groups with a seminormal Hall subgroup, Math. Notes. Vol. 80 (4), 2006. P. 542-549.

[9] W. Guo, Finite groups with seminormal Sylow subgroups, Acta Math. Sinica. Vol. $24(10)$, 2008. P. 1751-1758.

[10] V. N. Kniahina, V.S. Monakhov, Finite groups with semi-normal Schmidt subgroups, Algebra and Logic. Vol. 46 (4), 2007. P. 244-249.

[11] V.S. Monakhov, Introduction to the Theory of Finite groups and their Classes, Vyshejshaja shkola, 2006 (In Russian).

[12] Shemetkov, L. A. Formations of Finite Groups, M. : Nauka, 1978. [in Russian]

[13] V.S. Monakhov, Finite groups with abnormal and $\mathfrak{U}$-subnormal subgroups, Sib. Math. J. Vol. 57 (2), 2016. P. 353-363.

[14] B. Huppert, Endliche Gruppen I, Springer, 1967.

[15] V.S. Monakhov, Product of a biprimary and a 2-decomposable groups, Math. Notes, Vol. 23 (5), 1978. P. 355-359.

[16] J. H. Conway, R. T. Curtis, S. P. Norton, R. A. Parker, R. A. Wilson, Atlas of finite groups, London: Clarendon, 1985.

[17] V.S. Monakhov, Indices of maximal subgroups of finite soluble groups, Algebra and Logic. Vol. 43 (4), 2004. P. 230-237.

\section{CONTACT INFORMATION}

V. N. Kniahina,

V. S. Monakhov
Department of Mathematics, Francisk Skorina

Gomel State University, Sovetskaya str., 104,

Gomel 246019, Belarus

$$
\begin{aligned}
E \text {-Mail(s): Knyagina@inbox.ru, } \\
\text { Victor.Monakhov@gmail.com }
\end{aligned}
$$

Received by the editors: 23.04.2019. 